\title{
TWO CLASSICAL EXAMPLES OF GROSS' ABSTRACT WIENER MEASURE
}

\author{
HENRY C. FINLAYSON
}

\begin{abstract}
Classical Wiener measure on a space of functions of two variables and Wiener measure on the space of sample paths of two dimensional Brownian motion are shown to be special cases of Gross' abstract Wiener measure.
\end{abstract}

1. A construction has been given by Gross [3], [4] for an abstract Wiener measure on any real separable Banach space. In this construction the Banach space is expressed as the completion of a real separable Hilbert space $H$ with respect to a measurable norm. The measure is easily described once $H$ and the measurable norm are known.

Gross shows that a particular example is classical Wiener measure on the space $C$ of continuous functions on $[0,1]$ which vanish at zero. To do this he uses his theorem on measurable seminorms to establish the measurability of the supremum norm. In [2] is given a short direct proof of measurability of that norm by use of the Haar functions. It is the purpose of this paper to show, also by means of the Haar functions, that two other classical Wiener measures are special cases of abstract Wiener measure. The first is Wiener measure, established by Yeh [5], on the space $C_{2}$ of continuous functions on $[0,1] \times[0,1]$ which vanish on $[s, 0]$ and on $[0, t]$. The second is the space of sample paths of a two dimensional Brownian motion, i.e. the space of pairs of continuous functions $(x(t), y(t))$ on $[0,1]$ such that $(x(0)$, $y(0))=(0,0)$.

2. Description of $C_{2}$ as an abstract Wiener space. Here $H$ is the space of functions of the form

$$
\int_{0}^{t} \int_{0}^{s} \frac{\partial^{2} x(u, v)}{\partial u \partial v} d u d v
$$

such that $\partial^{2} x(u, v) / \partial u \partial v$ is square integrable on $[0,1] \times[0,1]$. The completion of $H$ in the uniform norm is $C_{2}$. In the lemma below, this norm is shown to be measurable, and thus $C_{2}$ is provided with abstract Wiener measure. That this measure agrees with that given by $[5,(2.2)$ and (2.3)] can be seen by consideration of suitable linear combinations of linear functionals on $C_{2}$ of the form $\int_{0}^{v} \int_{0}^{u} \rho\left(r, t_{i}\right) \rho\left(s, \tau_{j}\right) d r d s$ where

Received by the editors September 30, 1974 .

AMS (MOS) subject classifications (1970). Primary 28A40; Secondary 46G99. 


$$
\rho(s, t)= \begin{cases}0 & \text { if } 0 \leq s<t \\ 1 & \text { if } t \leq s \leq 1\end{cases}
$$

As in [2] the Haar functions are used in the computations in the lemma's proof. If $\left\{h_{i}(s)\right\}$ is the set of Haar functions on $[0,1]$ and if $B_{i}(t)=$ $\int_{0}^{t} h_{i}(s) d s$, then $\left\{B_{i}(s) B_{I}(t)\right\}$ is an orthonormal basis for $H$.

Lemma. For $\epsilon>0$ there exists $M>0$ such that

$$
\begin{aligned}
& {[1 / \sqrt{2 \pi}(K+L)(k+l)-(K-1)(k-1)]} \\
& \quad \cdot \int \ldots \int_{S} \exp \left[-\left(\sum_{i=k}^{k+l} \sum_{I=K}^{K+L}+\sum_{i=k}^{k+l} \sum_{I=1}^{K-1}+\sum_{i=1}^{k-1} \sum_{I=K}^{K+I}\right) \frac{x_{i I}^{2}}{2}\right] d x>1-\epsilon
\end{aligned}
$$

if $k, K>M$ where $\underline{x}=\left\{x_{i I}\right\}, d \underline{x}=\Pi_{i, I} d x_{i I}$, and

$$
S=\left\{\underline{x}: \sup _{s, t \in[0,1]}\left|\left(\sum_{i=k}^{k+l} \sum_{I=K}^{k+L}+\sum_{i=k}^{k+l} \sum_{I=1}^{K-1}+\sum_{i=1}^{k-1} \sum_{I=k}^{K+L}\right) x_{i I} B_{i}(s) B_{I}(t)\right|<\epsilon\right\} .
$$

Proof. It suffices to prove the lemma with $S$ replaced by the smaller set $S_{1} \cap S_{2} \cap S_{3}$ where

$$
S_{1}=\left\{x: \sup _{s, t \in[0,1]}\left|\sum_{i=k}^{k+l} \sum_{I=K}^{k+L} x_{i I} B_{i}(s) B_{I}(t)\right|<\frac{\epsilon}{3}\right\}
$$

and $S_{2}$ and $S_{3}$ are two obvious similar sets. Again it is sufficient to replace $S_{1}, S_{2}$ and $S_{3}$ by still smaller sets $T_{1}, T_{2}$ and $T_{3}$ in such a way that the integral over $T=T_{1} \cap T_{2} \cap T_{3}$ factors into integrals over $T_{1}, T_{2}$ and $T_{3} \cdot T_{1}$. is defined below and $T_{2}$ and $T_{3}$ are similar. If $B_{k}(s)$ and $B_{k+l}(s)$ are in the $n$th and $(n+q)$ th cycles of Haar functions, respectively, and $B_{K}(t)$ and $B_{K+L}(t)$ are in the $N$ th and $(N+Q)$ th cycles, then

$$
\sup _{s, t \in[0,1]}\left|\sum_{i=k}^{k+l} \sum_{I=K}^{K+!} x_{i I} B_{i}(s) B_{I}(t)\right| \leq \sum_{j=n}^{n+q} \sum_{J=i v}^{N+Q} \max _{\substack{i \in j \text { th cycle } \\ I \in J \text { th cycle }}} \frac{\left|x_{i i}\right|}{\left(4 \sqrt{2}^{j+J}\right)}
$$

Let

and restrict

$$
\Lambda=\sum_{j=1}^{\infty} \sum_{J:=1}^{\infty} 1 / \sqrt[4]{2}^{j+J}
$$

$$
\max _{\substack{i \in j \text { thcycle } \\ I \in J \text { th cycle }}}\left|x_{i I}\right| \text { by } \max _{\substack{i \in j \text { th cycle } \\ I \in J \text { th cycle }}} \frac{\left|x \sqrt{i I}^{j}\right|}{\sqrt{2^{j+J}}} \leq \frac{\epsilon}{\left(3 \sqrt[4]{2^{j+J}} \Lambda\right)} .
$$

That is, $T_{1}$ is given by

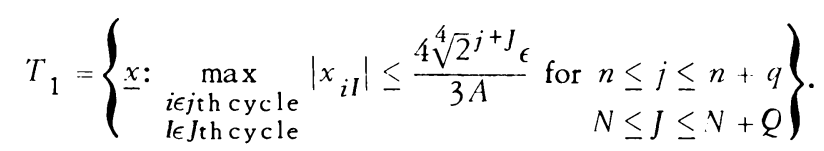


This restriction implies $\underline{x} \in S_{1}$ so that $T_{1} \subset S_{1}$. It is clear what $T_{2}$ and $T_{3}$ are and also that the integral factors into a product of integrals over $T_{1}$, $T_{2}$ and $T_{3}$. The factor of the integral over $T_{1}$ is greater than

$$
\begin{gathered}
\prod_{p=0, P=0}^{q, Q}\left[2 \int_{0}^{4^{4} \sqrt{2}^{n+p+N+P} \epsilon / 3 A} \frac{\exp \left(-u^{2} / 2\right) d u}{\sqrt{2 \pi}}\right] 2^{n+p+N+P} \\
=\prod_{p=0, P=0}^{q, Q}\left\{1-2\left[1-\ddot{\varphi}\left(4 \sqrt[4]{2}^{n+p+N+P} \epsilon / 3 A\right)\right]\right\}^{2^{n+p+N+P}}
\end{gathered}
$$

( $\Phi$ is the normal distribution; e.g. [1])

$$
\geq \prod_{p=0, P=0}^{q, Q}\left(1-a_{n+p+N+P}\right)^{2^{n+p+N+P}}
$$

where

$$
a_{j+k}=\left[6 A \exp \left(-16 \sqrt{2}^{j+k} \epsilon^{2} / 18 A^{2}\right)\right] /\left(4 \sqrt{2 \pi} \sqrt[4]{2}^{j+k} \epsilon\right)
$$

But

$$
\sum_{j, k=0}^{\infty} 2^{j+k} a_{j+k}=\sum_{n=0}^{\infty}(n+1) 2^{n} a_{n}
$$

converges so that

$$
\prod_{p=0, P=0}^{P, Q}\left(1-a_{n+p+N+P}\right)^{2^{n+p+N+P}}>\sqrt[3]{(1-\epsilon)}
$$

for $n$ or $N$ sufficiently large. Since either $n$ or $N$ large causes the product to be near one, it is easy to see that the integrals over $T_{2}$ and $T_{3}$ are also near one for both $n$ and $N$ sufficiently large and the proof is complete.

3. The space of sample paths of a two dimensional Brownian motion as an abstract Wiener space. In this case $H$ is the space of pairs of functions $\bar{x}(t)=\left(x_{1}(t), x_{2}(t)\right)$ such that $x_{1}^{\prime}(t)$ and $x_{2}^{\prime}(t)$ are square integrable on $[0,1]$. The inner product is given by

$$
(\bar{x}, \bar{y})=\int_{0}^{1}\left[x_{1}^{\prime}(t) y_{1}^{\prime}(t)+x_{2}^{\prime}(t) y_{2}^{\prime}(t)\right] d t .
$$

The norm, which will be shown to be measurable in the lemma below, is

$$
\|\bar{x}\|=\sup _{t \in[0,1]}\left|x_{1}(t)\right|+\sup _{t \in[0,1]}\left|x_{2}(t)\right|
$$

and the completion of $H$ in this norm is the space of pairs of continuous functions on $[0,1]$ which vanish at zero. Consideration of pairs of integrals of step functions for linear functionals will show that the abstract Wiener measure agrees with the transition function for the Brownian motion. The orthonormal basis used for $H$ is $A_{1}(t)=\left(B_{1}(t), 0\right), A_{2}(t)=\left(0, B_{1}(t)\right), A_{3}(t)$ $=\left(B_{2}(t), 0\right), A_{4}(t)=\left(0, B_{2}(t)\right)$, etc. 
Lemma. For $\epsilon>0$ there exists $M>0$ such that

$$
\left(\frac{1}{\sqrt{2 \pi}^{l+1}}\right) \int \ldots \int \exp \left[-\sum_{i=k}^{k+l} \frac{x_{i}^{2}}{2}\right] d \bar{x}>1-\epsilon
$$

if $l>M$ where $s=\left\{\bar{x}:\left\|\sum_{i=k}^{k+l} x_{i} A_{i}(t)\right\|<\epsilon\right\}$.

Proof. The proof will be given only for $k$ and $l$ odd. Then $\left\|\sum_{i=k}^{k+l} x_{i} A_{i}(t)\right\|$ is of the form

$$
\begin{aligned}
& \sup _{t \in[0,1]}\left|x_{k} B_{k}(t)+x_{k+2} B_{k+1}(t)+\cdots+x_{k+2 r} B_{k+r}(t)\right| \\
& \quad+\sup _{t \in[0,1]}\left|x_{k+1} B_{k}(t)+x_{k+3} B_{k+1}(t)+\cdots+x_{k+2 r+1} B_{k+r}(t)\right| \\
& \quad=\sup _{t \in[0,1]}\left|\sum_{i=k}^{k+r} x_{2 i-k} B_{i}(t)\right|+\sup _{t \in[0,1]}\left|\sum_{i=k}^{k+r} x_{2 i-k+1} B_{i}(t)\right| .
\end{aligned}
$$

Now if $B_{k}(t)$ and $B_{k+r}(t)$ are in the $n$th and $(n+q)$ th cycles, respectively, this sum is less than

$$
\sum_{j=n}^{n+q} \max _{i \in j \text { th cycle }} \frac{\left|x_{2 i-k}\right|}{2 \sqrt{2}^{j}}+\sum_{j=n}^{n+q} \max _{i \in j \text { th cycle }} \frac{\left|x_{2 i-k+1}\right|}{2 \sqrt{2}^{j}} .
$$

Let $K=\sum_{j=0}^{\infty} 1 / 2 \sqrt[4]{2}^{j}$ and impose the restriction

$$
\max _{i \in j \text { th cycle }} \frac{\left|x_{2 i-k}\right|}{2 \sqrt{2}^{j}} \leq \frac{\epsilon}{\left(4 \sqrt[4]{2}^{j} K\right)}, \quad \max _{i \in j \text { th cycle }} \frac{\left|x_{2 i-k+1}\right|}{2 \sqrt{2}^{j}} \leq \frac{\epsilon}{\left(4 \sqrt[4]{2}^{j} K\right)} .
$$

This restriction ensures that $\bar{x} \in S$. The integral is therefore greater than

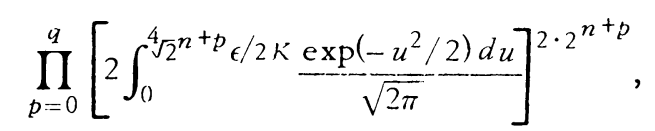

and since this product is again seen to converge, the proof is complete.

\section{REFERENCES}

1. W. Feller, An introduction to probability theory and its applications. Vol. 1, Wiley, New York, 1950. MR 12, 424.

2. H. C. Finlayson, Measurability of norm proved by Haar functions, Proc. Amer. Math. Soc. 53 (1975), 334-336.

3. L. Gross, Measurable functions on Hilbert space, Trans. Amer. Math. Soc. 105 (1962), 372-390. MR 26 \#5121.

4. - Abstract Wiener measure and infinite dimensional potential theory, Lectures in Modern Analysis and Applications, II, Lecture Notes in Math॰, vol. 140, Springer-Verlag, Berlin, 1970, pp. 84-116. MR 42 \#457.

5. J. Yeh, Wiener measure in a space of functions of two variables, Trans. Amer. Math. Soc. 95 (1960), 433-450. MR 23 \#A2735.

DEP ARTMENT OF MATHEMATICS, UNIVERSITY OF MANITOBA, WINNIPEG (19), MANITOBA, CAN ADA 